\title{
A Hybrid Method for Forecasting with an Introduction of a Day of the Week Index to the Daily Shipping Data of Sanitary Materials
}

\author{
Daisuke Takeyasu $^{1}$, Hirotake Yamashita ${ }^{2}$ and Kazuhiro Takeyasu ${ }^{3}$ \\ 1. Graduate School of Culture and Science, the Open University of Japan, 2-11 Wakaba, Mihama-District, Chiba City 261-8586, \\ Japan \\ 2. College of Business Administration and Information Science, Chubu University, 1200 Matsumoto-cho Kasugai, Aichi 487-8501, \\ Japan \\ 3. College of Business Administration, Tokoha University 325 Oobuchi, Fuji City, Shizuoka 417-0801, Japan
}

\begin{abstract}
Correct sales forecasting is inevitable in industries. In industries, how to improve forecasting accuracy such as sales, shipping is an important issue. There are many researches made on this. In this paper, we propose a new method to improve forecasting accuracy and confirm them by the numerical example. Focusing that the equation of ESM (exponential smoothing method) is equivalent to $(1,1)$ order ARMA model equation, a new method of estimation of smoothing constant in exponential smoothing method is proposed before by us which satisfies minimum variance of forecasting error. Generally, smoothing constant is selected arbitrarily. But in this paper, we utilize above stated theoretical solution. Firstly, we make estimation of ARMA model parameter and then estimate smoothing constants. Thus theoretical solution is derived in a simple way and it may be utilized in various fields. Combining the trend removing method with this method, we aim to improve forecasting accuracy. Furthermore, "a day of the week index" is newly introduced for the daily data and the forecasting is executed to the manufacturer's data of sanitary materials. We have obtained good result. The effectiveness of this method should be examined in various cases.
\end{abstract}

Key word: Minimum variance, exponential smoothing method, forecasting, trend, sanitary materials.

\section{Introduction}

Correct sales forecasting is inevitable in industries. Poor sales forecasting accuracy leads to increased inventory and prolonged dwell time of product. In order to improve forecasting accuracy, we have devised trend removal methods as well as searching optimal parameters and obtained good results. We created a new method and applied it to various time series and examined the effectiveness of the method. Applied data are sales data, production data, shipping data, stock market price data, flight passenger data etc.

Many methods for time series analysis have been

Corresponding author: Hirotake Yamashita, professor/MBA, research fields: supply chain management. E-mail: hr-yama@isc.chubu.ac.jp. presented such as AR Model (autoregressive model), ARMA Model (autoregressive moving average Model) and ESM (exponential smoothing method) [1-4]. Among these, ESM is said to be a practical simple method.

For this method, various improving method such as adding compensating item for time lag, coping with the time series with trend [5], utilizing Kalman Filter [6], Bayes Forecasting [7], adaptive ESM [8], exponentially weighted Moving Averages with irregular updating periods [9], making averages of forecasts using plural method [10] are presented. For example, Ref. [6] calculated smoothing constant in relationship with $\mathrm{S} / \mathrm{N}$ ratio under the assumption that the observation noise was added to the system. But he had to calculate under supposed noise because he 
couldn't grasp observation noise. It can be said that it does not pursue optimum solution from the very data themselves which should be derived by those estimation. Ref. [11] pointed out that the optimal smoothing constant was the solution of infinite order equation, but he did not show analytical solution. Based on these facts, we proposed a new method of estimation of smoothing constant in ESM before Ref. [12]. Focusing that the equation of ESM is equivalent to $(1,1)$ order ARMA model equation, a new method of estimation of smoothing constant in ESM was derived.

$$
\hat{x}_{t+1}=\sum_{l=0}^{\infty} \alpha(1-\alpha)^{l} x_{t-l}
$$

In this paper, utilizing above stated method, revised forecasting method is proposed. In making forecast such as shipping data, trend removing method is devised. Trend removing by the combination of linear and 2nd order non-linear function and 3rd order non-linear function is executed to the manufacturer's data of sanitary materials. The weights for these functions are varied by 0.01 increment and optimal weights are searched.

"A DWI (day of the week index)" is newly introduced for the daily data and a day of the week trend is removed. Theoretical solution of smoothing constant of ESM is calculated for both of the DWI trend removing data and the non DWI trend removing data. Then forecasting is executed on these data. This is a revised forecasting method. Variance of forecasting error of this newly proposed method is assumed to be less than those of the previously proposed method. The rest of the paper is organized as follows: In Section 2, ESM is stated by ARMA model and estimation method of smoothing constant is derived using ARMA model identification; The combination of linear and non-linear function is introduced for trend removing in Section 3; "DWI" is newly introduced in Section 4; Forecasting is executed in Section 5, and estimation accuracy is examined.

\section{Description of ESM Using ARMA Model}

In ESM, forecasting at time $t_{+1}$ is stated in the following equation.

$$
\begin{aligned}
\hat{x}_{t+1} & =\hat{x}_{t}+\alpha\left(x_{t}-\hat{x}_{t}\right) \\
& =\alpha x_{t}+(1-\alpha) \hat{x}_{t}
\end{aligned}
$$

Here,

$\hat{x}_{t+1}$ : forecasting at $t+1$;

$x_{t}:$ realized value at $t$;

$\alpha$ : smoothing constant $(0<\alpha<1)$;

Eq. (1) is re-stated as:

By the way, we consider the following $(1,1)$ order ARMA model.

$$
x_{t}-x_{t-1}=e_{t}-\beta e_{t-1}
$$

Generally, $(p, q)$ order ARMA model is stated as:

$$
x_{t}+\sum_{i=1}^{p} a_{i} x_{t-i}=e_{t}+\sum_{j=1}^{q} b_{j} e_{t-j}
$$

Here,

$\left\{x_{t}\right\}$ : Sample process of Stationary Ergodic Gaussian Process $x(t) t=1,2, \cdots, N, \cdots$;

$\left\{e_{t}\right\}$ : Gaussian White Noise with 0 mean $\sigma_{e}^{2}$ variance.

MA process in Eq. (4) is supposed to satisfy convertibility condition.

Utilizing the relation that:

$$
E\left[e_{t} \mid e_{t-1}, e_{t-2}, \cdots\right]=0
$$

We get the following equation from Eq. (3).

$$
\hat{x}_{t}=x_{t-1}-\beta e_{t-1}
$$

Operating this scheme on $t_{+1}$, we finally get:

$$
\begin{aligned}
\hat{x}_{t+1} & =\hat{x}_{t}+(1-\beta) e_{t} \\
& =\hat{x}_{t}+(1-\beta)\left(x_{t}-\hat{x}_{t}\right)
\end{aligned}
$$

If we set $1-\beta=\alpha$, the above equation is the same with (1), i.e., equation of ESM is equivalent to $(1,1)$ order ARMA model, or is said to be $(0,1,1)$ order ARIMA model because 1st order AR parameter is $-1([1,3])$. 
Comparing with Eq. (3) and Eq. (4), we obtain:

$$
\begin{aligned}
& a_{1}=-1 \\
& b_{1}=-\beta
\end{aligned}
$$

From Eqs. (1) and (6),

$$
\alpha=1-\beta
$$

Therefore, we get:

$$
\begin{aligned}
& a_{1}=-1 \\
& b_{1}=-\beta=\alpha-1
\end{aligned}
$$

From above, we can get estimation of smoothing constant after we identify the parameter of MA part of ARMA model. But, generally MA part of ARMA model become non-linear equations which are described below.

Let Eq. (4) be:

$$
\begin{aligned}
& \tilde{x}_{t}=x_{t}+\sum_{i=1}^{p} a_{i} x_{t-i} \\
& \tilde{x}_{t}=e_{t}+\sum_{j=1}^{q} b_{j} e_{t-j}
\end{aligned}
$$

We express the autocorrelation function of $\tilde{x}_{t}$ as $\tilde{r}_{k}$ and from Eqs. (8) and (9), we get the following non-linear equations which are well known [3].

$$
\begin{array}{lr}
\widetilde{r}_{k}=\sigma_{e}^{2} \sum_{j=0}^{q-k} b_{j} b_{k+j} & (k \leq q) \\
0 & (k \geq q+1) \\
\widetilde{r}_{0}=\sigma_{e}^{2} \sum_{j=0}^{q} b_{j}^{2} &
\end{array}
$$

For these equations, recursive algorithm has been developed. In this paper, parameter to be estimated is only $b_{1}$, so it can be solved in the following way.

From Eqs. (3), (4), (7) and (10), we get:

$$
\begin{aligned}
& q=1 \\
& a_{1}=-1 \\
& b_{1}=-\beta=\alpha-1 \\
& \widetilde{r}_{0}=\left(1+b_{1}^{2}\right) \sigma_{e}^{2} \\
& \widetilde{r}_{1}=b_{1} \sigma_{e}^{2}
\end{aligned}
$$

If we set:

$$
\rho_{k}=\frac{\widetilde{r}_{k}}{\widetilde{r}_{0}}
$$

the following equation is derived.

$$
\rho_{1}=\frac{b_{1}}{1+b_{1}^{2}}
$$

We can get $b_{1}$ as follows.

$$
b_{1}=\frac{1 \pm \sqrt{1-4 \rho_{1}^{2}}}{2 \rho_{1}}
$$

In order to have real roots, $\rho_{1}$ must satisfy:

$$
\left|\rho_{1}\right| \leq \frac{1}{2}
$$

From invertibility condition, $b_{1}$ must satisfy:

$$
\left|b_{1}\right|<1
$$

From Eq. (13), using the next relation,

$$
\begin{aligned}
& \left(1-b_{1}\right)^{2} \geq 0 \\
& \left(1+b_{1}\right)^{2} \geq 0
\end{aligned}
$$

Eq. (15) always holds.

As

$$
\alpha=b_{1}+1
$$

$b_{1}$ is within the range of:

$$
-1<b_{1}<0
$$

Finally we get:

$$
\begin{aligned}
& b_{1}=\frac{1-\sqrt{1-4 \rho_{1}^{2}}}{2 \rho_{1}} \\
& \alpha=\frac{1+2 \rho_{1}-\sqrt{1-4 \rho_{1}^{2}}}{2 \rho_{1}}
\end{aligned}
$$

which satisfy above condition. Thus we can obtain a theoretical solution by a simple way.

Here $\rho_{1}$ must satisfy:

$$
-\frac{1}{2}<\rho_{1}<0
$$

in order to satisfy $0<\alpha<1$.

Focusing on the idea that the equation of ESM is equivalent to $(1,1)$ order ARMA model equation, we can estimate smoothing constant after estimating ARMA model parameter.

It can be estimated only by calculating 0 th and 1 st order autocorrelation function. 


\section{Trend Removal Method}

As trend removal method, we describe the combination of linear and non-linear function.

(1) Linear function

We set:

$$
y=a_{1} x+b_{1}
$$

as a linear function.

(2) Non-linear function

We set:

$$
\begin{gathered}
y=a_{2} x^{2}+b_{2} x+c_{2} \\
y=a_{3} x^{3}+b_{3} x^{2}+c_{3} x+d_{3}
\end{gathered}
$$

as a 2 nd and a 3rd order non-linear function.

(3) The combination of linear and non-linear function

We set:

$$
\begin{aligned}
& y=\alpha_{1}\left(a_{1} x+b_{1}\right)+\alpha_{2}\left(a_{2} x^{2}+b_{2} x+c_{2}\right) \\
& +\alpha_{3}\left(a_{3} x^{3}+b_{3} x^{2}+c_{3} x+d_{3}\right) \\
& 0 \leq \alpha_{1} \leq 1 \quad, 0 \leq \alpha_{2} \leq 1 \quad, 0 \leq \alpha_{3} \leq 1 \\
& \alpha_{1}+\alpha_{2}+\alpha_{3}=1
\end{aligned}
$$

as the combination of linear and 2 nd order non-linear and 3rd order non-linear function.

Trend is removed by dividing the data by Eq. (21).

\section{A Day of the Week Index}

"DWI" is newly introduced for the daily data of sanitary materials. The forecasting accuracy would be improved after we identify the "a day of the week index" and utilize them. This time in this paper, the data we handle consist by Monday through Sunday,

we calculate $D W I_{j} \quad(j=1, \cdots, 7)$ for Monday through Sunday.

For example, if there is the daily data of $L_{\text {weeks }}$ as stated bellow:

$$
\left\{x_{i j}\right\}(i=1, \cdots, L)(j=1, \cdots, 7)
$$

Where, $x_{i j} \in R$ in which $L$ means the number of weeks (Here $L=10$ ), $i$ means the order of weeks
( $i$-th week), $j$ means the order in a week ( $j$-th order in a week; for example $j=1$ : Monday, $j=7$ : Sunday) and $x_{i j}$ is daily shipping data of sanitary materials. Then, $D W I_{j}$ is calculated as follows:

$$
D W I_{j}=\frac{\frac{1}{L} \sum_{i=1}^{L} x_{i j}}{\frac{1}{L} \cdot \frac{1}{7} \sum_{i=1}^{L} \sum_{j=1}^{7} x_{i j}}
$$

DWI trend removal is executed by dividing the data by Eq. (23). Numerical examples both of DWI removal case and non-removal case are discussed in Section 5.

\section{Forecasting the Sanitary Materials Data}

\subsection{Analysis Procedure}

The shipping data of 2 cases from January 31, 2012 to April 27, 2012 are analyzed. First of all, graphical charts of these time series data are exhibited in Figs. 1 and 2 .

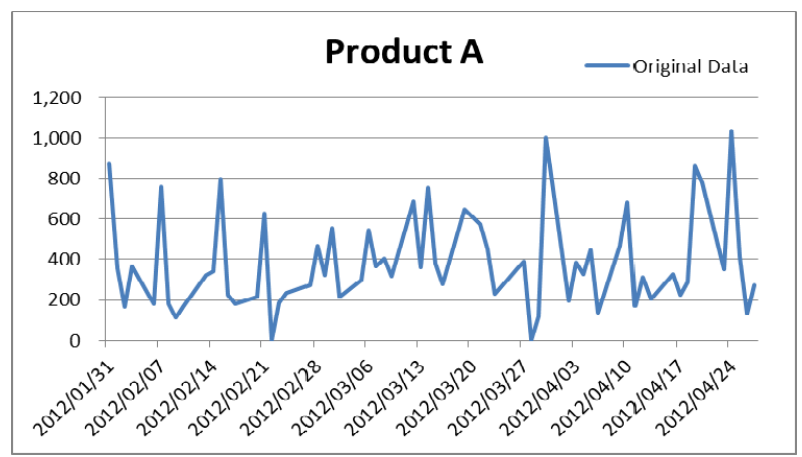

Fig. 1 Daily shipping data of product $A$.

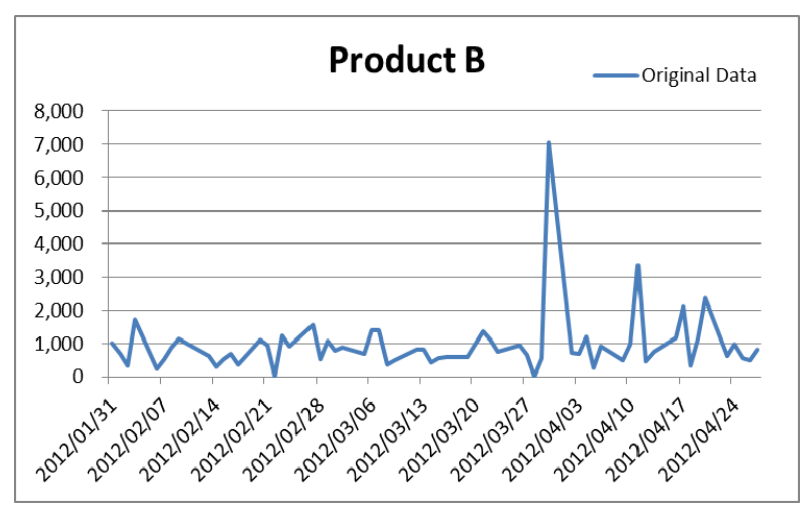

Fig. 2 Daily shipping data of product $B$. 


\section{A Hybrid Method for Forecasting With an Introduction of a Day of the Week Index to the Daily Shipping Data of Sanitary Materials}

Analysis procedure is as follows. There are 63 daily data for each case. We use 49 data (1 to 49) and remove trend by the method stated in Section 3. Then we calculate a DWI by the method stated in Section 4. After removing DWI trend, the method stated in Section 2 is applied and Exponential Smoothing Constant with minimum variance of forecasting error is estimated. Then 1 step forecast is executed. Thus, data is shifted to 2 nd to 50 th and the forecast for $51 \mathrm{st}$ data is executed consecutively, which finally reaches forecast of 63rd data. To examine the accuracy of forecasting, variance of forecasting error is calculated for the data of 50th to 63rd data. Final forecasting data is obtained by multiplying DWI and trend.

Forecasting error is expressed as:

$$
\begin{gathered}
\varepsilon_{i}=\hat{x}_{i}-x_{i} \\
\bar{\varepsilon}=\frac{1}{N} \sum_{i=1}^{N} \varepsilon_{i}
\end{gathered}
$$

Variance of forecasting error is calculated by:

$$
\sigma_{\varepsilon}^{2}=\frac{1}{N-1} \sum_{i=1}^{N}\left(\varepsilon_{i}-\bar{\varepsilon}\right)^{2}
$$

In this paper, we examine the two cases stated in Table 1.

\subsection{Trend Removing}

Trend is removed by dividing original data by Eq. (21). Here, the weight of $\alpha_{1}$ and $\alpha_{2}$ are shifted by 0.01 increment in Eq. (21) which satisfy the equation Eq. (22). The best solution is selected which minimizes the variance of forecasting error. Estimation results of coefficient of Eqs. (18)-(20) are exhibited in Table 2. Data are fitted to Eqs. (18)-(20), and using the least square method, parameters of Eqs. (18)-(20) are estimated. Estimation results of weights of Eq. (21) are exhibited in Table 3. The weighting parameter

As a result, we can observe the following three patterns.

(1) Selected liner model:

Product A Case 2, Product B Case 1

(2) Selected 2nd order model:

Product B Case 2,

(3) Selected 3rd order model:

Product A Case 1

Graphical charts of trend are exhibited in Figs. 3 and 4.

\subsection{Removing Trend by DWI}

After removing trend, a day of the week index is calculated by the method stated in Section 4. Calculation result for 1 st to 49 th data is exhibited in Table 4.

Table 1 The combination of the case of trend removal and DWI trend removal.

\begin{tabular}{lll}
\hline Case & Trend & DWI trend \\
\hline Case 1 & Removal & Removal \\
Case 2 & Removal & Non removal \\
\hline
\end{tabular}

Table 2 Coefficient of Eqs. (18)-(20).

\begin{tabular}{lllllllllll}
\hline & \multicolumn{3}{c}{ 1st } & \multicolumn{3}{c}{ 2nd } & \multicolumn{3}{c}{ 3rd } \\
& $a_{1}$ & $b_{1}$ & $a_{2}$ & $b_{2}$ & $c_{2}$ & $a_{1}$ & $a_{3}$ & $b_{3}$ & $c_{3}$ & $d_{3}$ \\
\hline Product A & 0.78 & 352.76 & 0.01 & 0.46 & 355.52 & 0.78 & -0.02 & 1.86 & -36.93 & 519.11 \\
Product B & 9.41 & 660.08 & 0.29 & -4.87 & 781.45 & 9.41 & -0.02 & 2.09 & -41.29 & 940.79 \\
\hline
\end{tabular}

Table 3 Weights of Eq. (21).

\begin{tabular}{lllll}
\hline & Case & $\alpha_{1}$ & $\alpha_{2}$ & $\alpha_{3}$ \\
\hline \multirow{2}{*}{ Product A } & Case 1 & 0.86 & 0.09 & 0.05 \\
& Case 2 & 1.00 & 0.00 & 0.00 \\
\hline \multirow{2}{*}{ Product B } & Case 1 & 1.00 & 0.00 & 0.00 \\
& Case 2 & 0.95 & 0.05 & 0.00 \\
\hline
\end{tabular}



Week Index to the Daily Shipping Data of Sanitary Materials

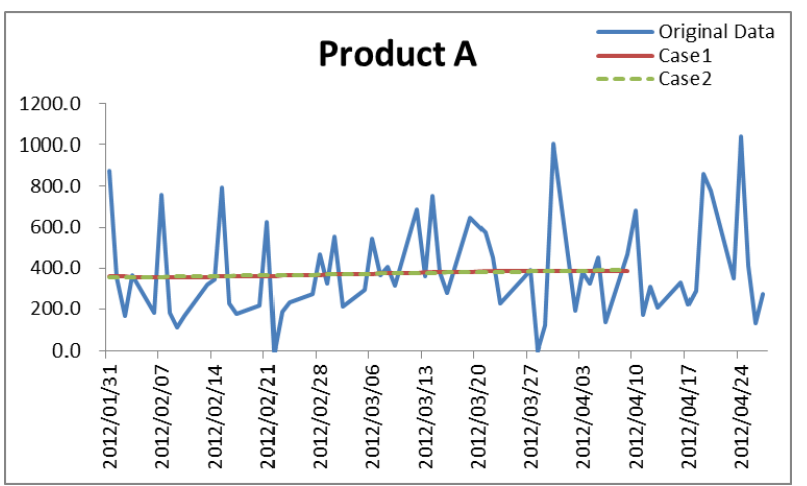

Fig. 3 Daily shipping data of product $A$.

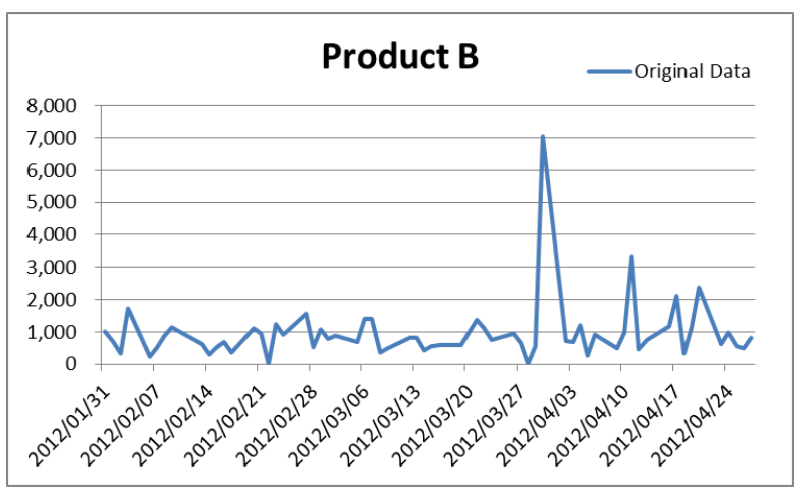

Fig. 4 Daily shipping data of product $B$.

\subsection{Estimation of Smoothing Constant with Minimum} Variance of Forecasting Error

After removing DWI trend, Smoothing Constant with minimum variance of forecasting error is estimated utilizing Eq. (16). There are cases that we cannot obtain a theoretical solution because they do not satisfy the condition of Eq. (17). In those cases, Smoothing Constant with minimum variance of forecasting error is derived by shifting variable from 0.01 to 0.99 with 0.01 interval. Calculation result for
1 st to 49th data is exhibited in Table 5.

\subsection{Forecasting and Variance of Forecasting Error}

Utilizing smoothing constant estimated in the previous section, forecasting is executed for the data of 50th to 63rd data. Final forecasting data is obtained by multiplying DWI and trend. Variance of forecasting error is calculated by Eq. (26).

Forecasting results are exhibited in Figs. 5 and 6

Variance of forecasting error is exhibited in Table 6.

\section{Conclusions}

Correct sales forecasting is inevitable in industries. Focusing on the idea that the equation of ESM was equivalent to $(1,1)$ order ARMA model equation, a new method of estimation of smoothing constant in exponential smoothing method was proposed before by us which satisfied minimum variance of forecasting error. Generally, smoothing constant was selected arbitrarily. But in this paper, we utilized above stated theoretical solution. Firstly, we made estimation of ARMA model parameter and then estimated smoothing constants. Thus theoretical solution was derived in a simple way and it might be utilized in various fields.

Furthermore, combining the trend removal method with this method, we aimed to increase forecasting accuracy. An approach to this method was executed in the following method. Trend removal by a linear function was applied to the daily shipping data of sanitary materials. The combination of linear and

Table 4 A day of the week index.

\begin{tabular}{lllllllll}
\hline \multirow{2}{*}{ Airline } & \multirow{2}{*}{ Case } & \multicolumn{7}{c}{ A day of the week index } \\
\cline { 3 - 9 } & & Thu. & Fri. & Sat. & Sun. & Mon. & Tue. & Wed. \\
\hline A & Case1 & 1.308 & 1.170 & 0.638 & 1.004 & 1.144 & 0.797 & 0.939 \\
B & Case1 & 2.017 & 1.014 & 0.647 & 1.087 & 0.725 & 0.905 & 0.605 \\
\hline
\end{tabular}

Table 5 Estimated smoothing constant with minimum variance.

\begin{tabular}{llll}
\hline & Case & $\rho_{1}$ & $\alpha$ \\
\hline \multirow{2}{*}{ Product A } & Case 1 & -0.1309 & 0.87 \\
& Case 2 & -0.1481 & 0.85 \\
\hline \multirow{2}{*}{ Product B } & Case 1 & -0.0932 & 0.91 \\
& Case 2 & -0.1089 & 0.89 \\
\hline
\end{tabular}



Week Index to the Daily Shipping Data of Sanitary Materials

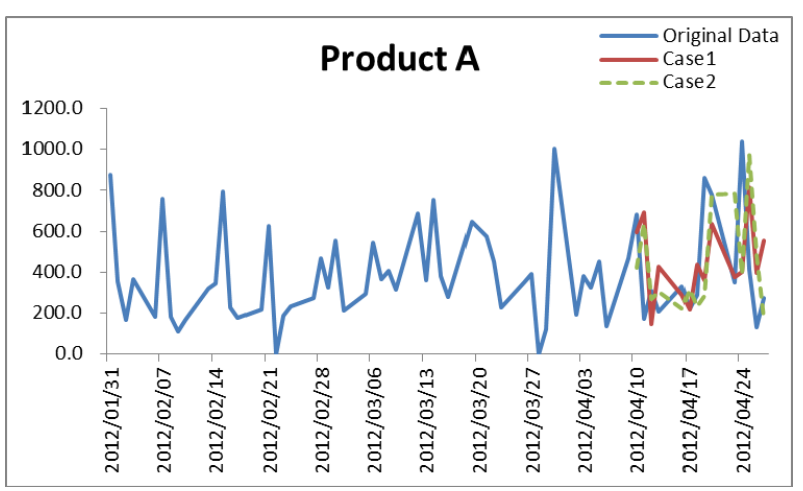

Fig. 5 Forecasting results of product $A$.

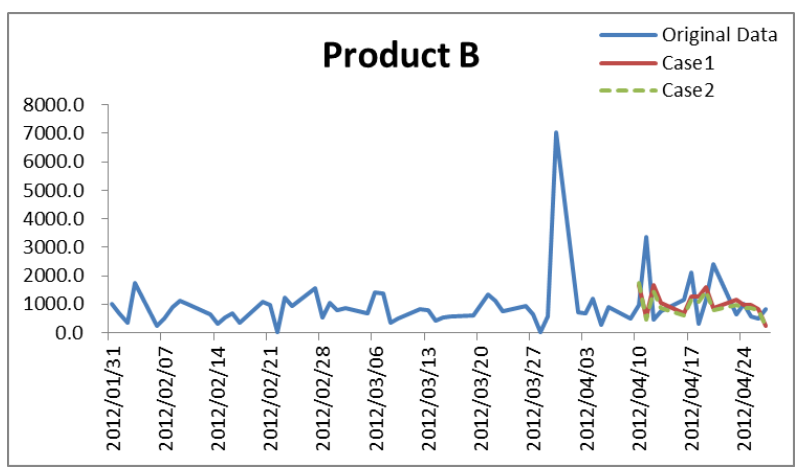

Fig. 6 Forecasting results of product $B$.

Table 6 Variance of forecasting error.

\begin{tabular}{llll}
\hline & Case & Variance of Forecasting Error & \\
\hline \multirow{2}{*}{ Product A } & Case 1 & $39,904.97$ & $*$ \\
& Case 2 & $52,292.11$ & \\
\multirow{2}{*}{ Product B } & Case 1 & $492,514.45$ & $*$ \\
& Case 2 & $755,371.72$ & \\
\hline
\end{tabular}

non-linear function was also introduced in trend removing. "DWI" is newly introduced for the daily data and a day of the week trend is removed. Theoretical solution of smoothing constant of ESM was calculated for both of the DWI trend removing data and the non DWI trend removing data. Then forecasting was executed on these data.

Regarding both data, the forecasting accuracy of case 1 (DWI is imbedded) was better than those of case 2 (DWI is not imbedded). It can be said that the introduction of DWI has worked well. It is our future works to ascertain our newly proposed method in many other cases. The effectiveness of this method should be examined in various cases.

In the end, we appreciate Mr. Norio Funato for his helpful support of our study.

\section{References}

[1] Jenkins, B. 1994. Time Series Analysis Third Edition. Prentice Hall.

[2] Brown, R. G. 1963. Smoothing, Forecasting and Prediction of Discrete -Time Series. Prentice Hall.

[3] Tokumaru, H. 1982. Analysis and Measurement-Theory and Application of Random Data Handling. Baifukan Publishing.

[4] Kobayashi, K. 1992. Sales Forecasting for Budgeting. Chuokeizai-Sha Publishing.

[5] Winters, P. R. 1984. "Forecasting Sales by Exponentially Weighted Moving Averages." Management Science 6: 324-43.

[6] Maeda, K. 1984. "Smoothing Constant of Exponential Smoothing Method." Seikei University Report Faculty of Engineering: 2477-84.

[7] West, M., and Harrison, P. J. 1989. Baysian Forecasting and Dynamic Models. New York: Springer-Verlag.

[8] Ekern, S. 1982. "Adaptive Exponential Smoothing Revisited." Journal of the Operational Research Society 32: 775-82.

[9] Johnston, F. R. 1993. "Exponentially Weighted Moving Average (EWMA) with Irregular Updating Periods." Journal of the Operational Research Society 44: 711-6.

[10] Makridakis, S., and Winkler, R. L. 1983. "Averages of Forecasts; Some Empirical Results." Management Science 29: 987-96.

[11] Ishii, N. 1991. "Bilateral Exponential Smoothing of Time Series.” Int.J.System Sci. 12: 997-88.

[12] Takeyasu, K., and Nagao, K. 2008. "Estimation of Smoothing Constant of Minimum Variance and Its Application to Industrial Data." Industrial Engineering and Management Systems 7: 44-50. 